\title{
Enhanced hyaluronic acid fat grafts, histological findings and specific markers
}

\begin{abstract}
Fat grafts have become particularly popular in recent years due to their autologous origin, permanent volumetric and regenerative properties. Different attempts in improving the re-absorption rate through manipulation or enhancement are possible.

Hyaluronic acid is one of the agents that could improve the outcome of the treatment given its natural anticoagulant and volume stabilizer actions. A new HA dermal filler Intraline 1 (Intraline, BC, Canada) was used to enhanced the fat grafts prior to inoculation.

Samples of the enhanced grafts were extracted for laboratory analysis, cytometry, staining and markers analysis. Control fat grafts were taken, to compare the results.

Histologic findings show better cytoarchitecture, less cellular rupture and inflammation in the HA enhanced fat graft.

The former findings show that fat graft enhancement through HA can be a promising way to potentiate the action of the graft prior to inoculation.
\end{abstract}

Volume I Issue I - 2017

\section{Torres S, Ruminot C, Nuciforo G, Bonanno} C

Dermatology \& Cosmetology, Aesthetic surgery private practice, Italy

Correspondence: Sebastian Torres, Dermatology \& Cosmetology, Aesthetic surgery private practice, via Caposile 2, Rome, Italy-00195, Tel No +39 3928833702. Email storres 100@gmail.com

Received:September 15, 2016 | Published: March 24, 2017

\section{Introduction}

Fat grafting has become one of the main techniques in aesthetic and reconstructive surgery due to its easy availability, autologous origin, volumetric and regenerative capacities. Within the graft, transplanted cells experiment a constant effort in establishing a vital support from the surrounding environment that will allow a definitive integration. Only a fraction will accomplish the former, the rest following death and reabsorption.

Different attempts in improving the reabsorption rate through manipulation or enhancement are possible. The list include the use of platelet-rich plasma, ${ }^{2}$ adipose-derived stem cells ex vivo expansion, ${ }^{3}$ adipose-derived stem cells filters, local pretreatment with botulinum toxin, ${ }^{4}$ only to name few. Hyaluronic acid is one of the agents that could improve the outcome of the treatment given its natural anticoagulant and volume stabilizer actions.

\section{Materials and methods}

Private aesthetic patients who had requested liposuction were included in the study. A specific informed consent, properly discussed and signed, allow obtaining tissue samples for processing and analysis.

Donor site anesthesia with lidocaine low-level tumescent solution was employed.

Fat graft samples were manually taken with the aid of Tonnard harvesting cannula, $2 \mathrm{mmx} 10 \mathrm{~cm}, 0.8 \mathrm{~mm}$ port apertures (Tulip Medical, San Diego, CA, USA), and 20ml Vacklock syringe (Shippert medical, Denver, CO, USA). Fat processing ringer lactate washing was used to eliminate oil and residues.

Fat grafts were fatherly prepared with a mix of $20 \%$ new cross linked hyaluronic acid dermal filler, Intraline 1 (Intraline, $\mathrm{BC}$, Canada). This new hyaluronic acid dermal filler has a spherification technology that allows smooth displacement of the HA molecules and a uniform mix with fat parcels. The solution was adequately mix through a new emulsifier device (Tulip Medical, San Diego, CA, USA). Control graft with no HA mix were kept and emulsified accordingly. Finally, the samples were immerse in cellular stabilizing (fixing) solution prior to histological processing.

The samples were centrifuged at $2150 \mathrm{rpm}$ for 10 minutes. Successively, part of the supranadant was extracted with a Pasteur pipet and depending on quantity was; re-suspended and slide slithered (if scarse) or agar included (cyto-inclusion) if abundant and processed to obtain a paraffin block that was cut to obtain the necessary slides for the study. Invisible pellets were re-suspended in adhesion facilitating liquid, prior to cyto-inclusion.

Hematoxilin/Eosin staining and inmunohistochemical analysis (S100-CD31, etc) was done prior to pathologist observation.

\section{Results}

Histologic findings show cohesive, variable size adipocytes with good morphology and scarse fibrosis and inflammation in the HA-Fat mix group (Figure 1).

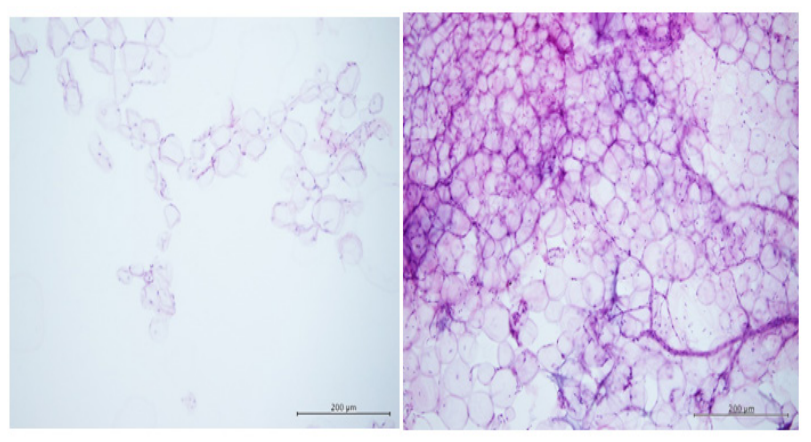

Figure I Supernatant histological samples of Fat isolated (right) and Fat-HA (left) groups.A greater number of adipocytes clusters is seen in the latter. 
Control group evidenced isolated or small adipocytes clusters, some of them with regressive degeneration. Intensification of support vascular fibers and rare inflammatory infiltrates were visible.

The supranatant of the samples was tested through immune cyto chemistry with S100. This marker showed greater integrity of the adipocytes in the HA mix group and lesser swelling (Figures 2).

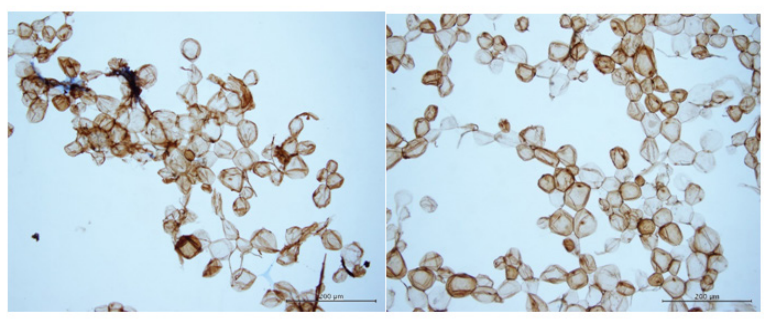

Figure 2 Supernatant histological samples S-100 marker of Fat isolated (right) and Fat-HA (left) group. Notice the better morphology of the adipocytes and lesser inflammation on the latter.

The sediment was analyzed to observe the presence of flogosis and vascular representation. Inflammation was minimal in both groups, but vascular component was greater in the Fat-HA group (Figure 3). Overall the study group presents better cyto architecture, less cellular rupture and inflammation than the controls.

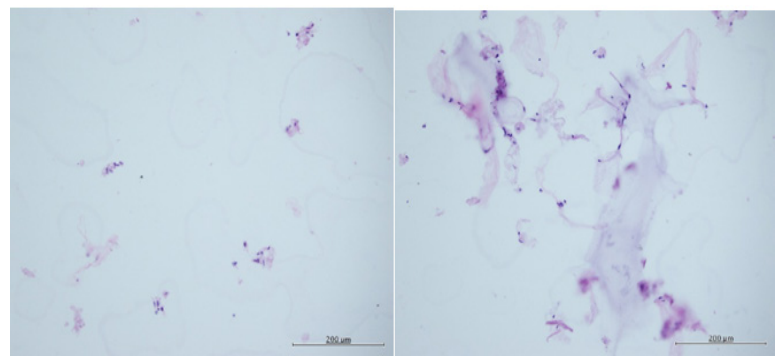

Figure 3 Sediment histological samples of Fat isolated (right) and Fat-HA (left) group that allows observation of flogosis and vascular representation. Both samples show scarce inflammation but the latter evidenced greater vascular component.

\section{Discussion}

The association of hyaluronic acid and fat have been used in different fields of medicine. In tympanic perforations, the combination therapy has demonstrated better outcomes than either isolated substance ${ }^{5-7}$ comparable to gold standard surgical closure. ${ }^{8}$ It has been proposed in this clinical setting, that the association of HA and fat, increases endogenous growth factors expression though accelerating healing. ${ }^{9}$ Prostate cancer patient treated through radio brachytherapy frequently experiment gastrointestinal disorders. In this group, HA pretreatment of the perianal fat disminish GI toxicity. ${ }^{10}$

HIV related facial lipodystrophy treated with HA subcutaneous injections increased the thickness Injection of hyaluronic acid in facial fat regions atrophy in patients with HIV cutaneous demonstrated increased cutaneous and subcutaneous thickness Eco graphically, with a response rate of $60 \%$ and follow up to 52 weeks. ${ }^{11}$

In vitro studies have shown that adipogenesis can be down regulated through HA synthesis inhibition. Furthermore In vivo animals studies have corroborated this premise, where abdominal fat accumulation was suppressed by exogenous hyaluronidase sumministration. The former was associated with a reduction of fat liver deposits and increase in insulin sensitivity. According to this, extracellular matrix HA variations, are crucial for adipogenesis. ${ }^{12}$

Fat grafting outcomes rely on the primary integration of transplant adipocytes and regenerative properties of adipose stem cells which will help to reestablish a new normal connective tissue through a new vascular bed. Within the graft, transplanted cells experiment a constant effort in establishing a vital support from the surrounding environment that will allow a definitive integration. Only a fraction will accomplish the former, the rest following death and re-absorption.

Different attempts in improving the re-absorption rate through manipulation or enhancement are possible through systemic or local measures. The former includes oxygen therapy, ischemia limiting actions and oral supplements. Local enhancement techniques include the use of platelet-rich plasma, ${ }^{2}$ adipose-derived stem cells ex vivo expansion, ${ }^{3}$ adipose-derived stem cells filters and local pretreatment with botulinum toxin, ${ }^{4}$ among others.

Hyaluronic acid is one of the agents that could improve the outcome of the treatment given its natural anticoagulant and volume stabilizer actions. ${ }^{13}$ The former property may be especially useful in the first week after the graft maintaining a fluid micro circulation that may enhance neoangiogenesis. Moreover given its volumetric stabilizing capacity and natural water bonding tendencies, could stabilize the hydrostatic and osmotic pressure within the adipocytes, impending cellular dehydration, damage and rupture.

HA enhancement of fat grafts generates a soft emulsion of lesser viscocity allowing it to flow through smaller delivering devices and reducing the mechanical stress of the graft at the delivery point. Moreover HA competes with water within the aspirate material and displaces it, maintaining cellular cohesiveness. The former findings show that fat graft enhancement through HA can be a promising way to potentiate the action of the graft prior to inoculation.

\section{Acknowledgements}

None.

\section{Conflict of interest}

The author declares no conflict of interest.

\section{References}

1. Eto H, Kato H, Suga H, et al. The fate of adipocytes after nonvascularized fat grafting: evidence of early death and replacement of adipocytes. Plast Reconstr Surg. 2012;129(5):1081-1092.

2. Sommeling CE, Heyneman A, Hoeksema H, et al. The use of platelet-rich plasma in plastic surgery: a systematic review. $J$ Plast Reconstr Aesthet Surg. 2013;66(3):301-311.

3. TrojahnKolle SF, Oliveri RS, Glovinski PV, et al. Importance of mesenchymal stem cells in autologous fat grafting: a systematic review of existing studies. J Plast Surg Hand Surg. 2012;46(2):59-68.

4. Baek RM, Park SO, Jeong EC, et al. The effect of botulinum toxin a on fat graft survival. Aesthetic Plast Surg. 2012;36(3):680-686.

5. Saliba I. Hyaluronic acid fat graft myringoplasty: how we do it. Clin Otolaryngol. 2008;33(6):610-614.

6. Alzahrani M, Saliba I. Hyaluronic acid fat graft myringoplasty vs fat patch fat graft myringoplasty. Eur Arch Otorhinolaryngol. 2015;272(8):1873-1877. 
7. Prior M, Gibbins N, John G, et al. Hyaluronic acid ester in myringoplasty. J Laryngol Otol. 2008;122(2):e3.

8. Saliba I, Woods O. Hyaluronic acid fat graft myringoplasty: a minimally invasive technique. Laryngoscope. 2011;121(2):375-380.

9. Saliba I, Alzahrani M, Zhu T, et al. Growth factors expression in hyaluronic acid fat graft myringoplasty. Laryngoscope. 2014;124(6):E224-230.

10. Prada PJ, Jimenez I, Gonzalez-Suarez H, et al. High-dose-rate interstitial brachytherapy as monotherapy in one fraction and transperineal hyaluronic acid injection into the perirectal fat for the treatment of favorable stage prostate cancer: Treatment description and preliminary results. Brachytherapy. 2012;11(2):105-110.
11. Bugge H, Negaard A, Skeie L, et al. Hyaluronic acid treatment of facial fat atrophy in HIV-positive patients. HIV Med. 2007;8(8):475-482.

12. Ji E, Jung MY, Park JH, et al. Inhibition of adipogenesis in 3T3-L1 cells and suppression of abdominal fat accumulation in high-fat diet-feeding C57BL/6J mice after downregulation of hyaluronic acid. Int J Obes (Lond). 2014;38(8):1035-1043.

13. Alghoul M, Mendiola A, Seth R, et al. The effect of hyaluronan hydrogel on fat graft survival. AesthetSurg J. 2012;32(5):622-633. 